Applied and

NISTIR 90-4389

Computational

Mathematics

Division

Center for Computing and Applied Mathematics

The Effect of Gravity Modulation on Solutal Convection during Directional Solidification

\author{
B.T. Murray, S.R. Coriell, \\ and G.B. McFadden
}

August 1990

U.S. DEPARTMENT OF COMMERCE

National Institute of Standards and Technology

Gaithersburg, MD 20899 



\section{THE EFFECT OF GRAVITY MODULATION ON SOLUTAL CONVECTION DURING DIRECTIONAL SOLIDIFICATION}

\author{
B. T. Murray \\ S. R. Coriell \\ G. B. McFadden
}

U.S. DEPARTMENT OF COMMERCE Natlonal Institut $\theta$ of Standards and Technology Center for Computing and Applled Mathematics Applled Computatlonal Mathematics Divislon

Galthersburg, MD 20899

August 1990

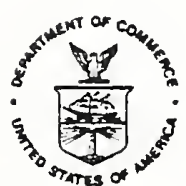

John W. Lyons, Director 
. 


\title{
The Effect of Gravity Modulation on Solutal Convection during Directional Solidification
}

\author{
B. T. Murray, S. R. Coriell, and G. B. McFadden \\ National Institute of Standards and Technology \\ Gaithersburg, Maryland 20899, USA
}

\begin{abstract}
During directional solidification of a binary alloy at constant velocity, thermosolutal convection may occur due to the temperature and solute gradients associated with the solidification process. For vertical growth in an ideal furnace (lacking horizontal gradients) a quiescent state is possible. For a range of processing conditions, the thermal Rayleigh number is sufficiently small that the stabilizing role of the thermal field during growth vertically upwards may be neglected, and only solutal convection need be considered. The effect of a time-periodic vertical gravitational acceleration (or equivalently vibration) on the onset of solutal convection is calculated based on linear stability using Floquet theory. We find that a stable base state can be destabilized due to modulation, while an unstable state can be stabilized. The flow and solute disturbance fields show both synchronous and subharmonic temporal response to the driving sinusoidal modulation.
\end{abstract}




\section{Introduction}

During solidification of a binary alloy, temperature and solute gradients are inherently present, and cause a density gradient in the melt. The action of a gravitational field on this density gradient can give rise to fluid flow in the melt with consequent redistribution of solute, which may result in an undesirable solute distribution. The possibility of processing materials in space is attractive, since the low level background gravitational acceleration $\left(10^{-6}\right.$ of the earth's gravitational acceleration, $\left.g_{e}\right)$ can potentially eliminate buoyancy-driven convection. However, owing to orbital maneuvers and inherent mechanical vibrations, the occurrence of time-dependent local acceleration ( $\mathrm{g}$-jitter) may by itself induce buoyant convection. The local residual accelerations in space laboratories have been characterized $[1,2]$. On earth vibration may be useful as a means for controlling convection. This idea has been investigated recently for two systems $\left(\mathrm{CsCdCl}_{3}[3]\right.$ and $\left.\mathrm{CdTe}[4]\right)$, where low frequency vibrations were used in an effort to control the interface shape and position during Bridgman growth.

For vertical Bridgman growth, the growth direction is aligned with the gravity vector. Ideally, only the vertical thermal and solutal gradients from the solidification process exist

and a quiescent state in the melt is possible. For growth of a pure material vertically upwards, the temperature will increase with height, and for a normal melt which expands on heating the density will decrease with height. Hence, for growth vertically upwards the temperature field is stabilizing with respect to convective instabilities. For an alloy which rejects a light solute upon solidification, the solute gradient which develops above the interface tends to increase the density of the melt with height. As a result of this situation, either solutal or thermosolutal convection may occur depending on the relative magnitude of the temperature and concentration gradients.

The onset of thermosolutal convection during directional solidification in a constant gravitational field was studied by Coriell et al. [5], and the onset of solutal convection alone by Hurle et al. [6]. The review article by Glicksman et al. [7] gives a comprehensive bibliography on convection in directional solidification, which includes studies of the related interfacial in- 
stability problem as well as the interaction of convective and interfacial instabilities. Results from these previous studies indicate that for dilute alloys under processing conditions where interfacial instabilities should not occur, it may be very difficult to avoid convection under terrestrial conditions. In order to evaluate the practicality of space-based processing using the Bridgman technique, the effect of local time-dependent residual accelerations on buoyant convection must be investigated. A recent review by Alexander [8] discusses the effect of residual accelerations on heat and mass transfer in low-gravity materials experiments. The concern of the present study is whether sinusoidal time-dependent accelerations can lead to the onset of convection in a quiescent melt when the accelerations are aligned with the growth direction.

The stability of fluid systems undergoing time-periodic forcing has been reviewed by Davis [9] and Ostrach [10]. The bulk of the existing work has concentrated on RayleighBenard convection subject to gravity modulation or boundary temperature modulation, and Taylor-Couette flow with time-periodic torsional oscillation of the cylinders. Some early work on temporally modulated Rayleigh-Benard convection by Gershuni and Zhukhovitskii is summarized in their book on convective stability [11]. They considered different aspects of time-periodic modulation, including Floquet theory for the linear onset conditions and finite-difference calculations of the nonlinear behavior for sinusoidal modulation. They found that both stabilization and destabilization can be obtained as a result of the modulation. They also employed averaging techniques to study the asymptotic behavior in the case of high frequency modulation. For Rayleigh-Benard convection subject to sinusoidal gravitational acceleration, Gresho and Sani [12] performed an extensive study of the linear theory parameter space using Floquet analysis and a highly truncated Galerkin expansion for the disturbance solutions. In the extreme case of a single expansion function, the analysis is reduced to consideration of the stability behavior of the Mathieu equation. They found that sinusoidal gravity modulation has a significant effect on the stability limits of RayleighBenard convection. Disturbance response having the same frequency as the modulation (synchronous behavior) is found for low to moderate modulation frequencies, while response 
with half the modulation frequency (subharmonic behavior) is obtained for high frequency modulation. In addition to linear theory, they studied the nonlinear behavior resulting from sinusoidal modulation using a low-order Galerkin expansion.

More recently, Wadih and Roux [13] have considered the effect of gravity modulation on an infinite vertical cylinder of fluid subject to a constant vertical temperature gradient. A three-dimensional simulation of Rayleigh-Benard convection with gravitational modulation has been performed by Biringen and Peltier [14]. Nonlinear convection was simulated re-

sulting from both sinusoidal and random modulation with and without a one- $g$ background. They obtained synchronous and subharmonic response resulting from sinusoidal modulation in agreement with Gresho and Sani, and showed how the finite-amplitude solutions transport heat under various modulation conditions.

In the following section, the simplified model of solutal convection in directional solidification is presented. The emphasis of the present work is the conditions for the onset of convection from the quiescent state based on linear stability theory. Because of the periodic time-dependent forcing, Floquet theory is used in the stability analysis. The solution for the onset conditions requires numerical solutions of the resulting differential eigenvalue problem. Two distinct numerical approaches are used to obtain the linear stability solutions. Results for a range of parameters corresponding to different alloy systems are presented. Some brief results from nonlinear calculations are included to elucidate the linear results and provide an additional verification of the linear theory results.

\section{Governing equations}

The model for solutally-driven buoyant convection in directional solidification is shown in Fig. 1. The exponential solute profile in the melt results from the rejection of solute at the interface. Depending on the densities of the alloy constituents and the growth direction, a statically unstable density stratification may result from the concentration variation. For steady-state conditions, the solid composition is equal to the far-field concentration in the liquid $c_{\infty}$. The solute jump at the interface is characterized by the equilibrium segregation 
coefficient $k$, which is the ratio of the solid and liquid concentrations at the interface; for $k$ leśs than one, solute is rejected at the interface. For growth vertically upwards, rejection of a light solute produces the unstable stratification. For the present study, the effects of the thermal field and deformation of the interface will not be included, but are part of an ongoing investigation of this problem. We also assume that the densities of the crystal and the melt are equal, thus neglecting the possibility of convection due to a density change.

In order to investigate the onset and development of buoyancy-driven solutal convection during directional solidification, the governing equations are written in terms of a Cartesian coordinate system $(x, y, z)$ which is attached to the planar crystal-melt interface moving at constant growth velocity $V$ in the positive $z$ direction. The fluid velocity in the melt is described by the vector field $\boldsymbol{u}=(u, v, w)$. This velocity is measured in the laboratory frame in which the crystal is at rest, so that in the undisturbed state $\boldsymbol{u}=0$. Assuming an incompressible Newtonian Aluid, the basic governing equations for the problem are the conservation of mass equation, the Navier-Stokes equations written in vector form, the convection-diffusion equation for the solute concentration $c$, and a linear equation of state expressing the dependence of the melt density $\rho$ on solute concentration,

$$
\begin{gathered}
\nabla \cdot \boldsymbol{u}=0 \\
\frac{\partial \boldsymbol{u}}{\partial t}+\left(\boldsymbol{u}^{*} \cdot \nabla\right) \boldsymbol{u}=-\nabla p / \rho_{0}+\nu \nabla^{2} \boldsymbol{u}+\boldsymbol{g} \rho / \rho_{0} \\
\frac{\partial c}{\partial t}+\left(\boldsymbol{u}^{*} \cdot \nabla\right) c=D \nabla^{2} c \\
\rho=\rho_{0}\left[1-\beta\left(c-c_{0}\right)\right]
\end{gathered}
$$

where the velocity $\boldsymbol{u}^{*}=\boldsymbol{u}-(0,0,-V)$ results from fixing the reference frame to the moving solid-liquid interface. Here $p$ is the fluid pressure, $\nu$ is the kinematic viscosity, $D$ is the solute diffusion coefficient, and $\beta$ is the solutal expansion coefficient. The Boussinesq approximation is employed which assumes that density variation is important only in the body force term in the Navier-Stokes equations. The remaining fluid parameters $(\nu, D$, and $\beta)$ are assumed constant. The subscript zero refers to reference values of the parameters. 
For a gravitational field aligned with the growth direction, a motionless solution exists. We consider the linear stability of this motionless base state subject to time-periodic gravitational acceleration. The model for solutal convection during directional solidification assumes growth with a rigid planar interface in the presence of a vertical gravitational field that varies sinusoidally in time according to $g(t)=g_{0}+g_{1} \cos (\bar{\Omega} t)$, where $\bar{\Omega}$ is the dimensional forcing frequency. The liquid is assumed to be semi-infinite in the vertical $(z)$ direction, and laterally unbounded so that solutions that are periodic in the horizontal directions are possible. The steady-state base solution consists of no fluid motion and the exponential solute profile obtained from solving the steady, one-dimensional diffusion equation in the semi-infinite liquid region subject to solute conservation at the growing interface, given by

$$
D \frac{\partial c}{\partial z}=-V(1-k) c
$$

and subject to the far-field concentration value $c_{\infty}$.

For the linear stability analysis of the base state, the flow field variables are written as the superposition of the base state component and a perturbation. The perturbed quantities are Fourier analyzed in the lateral directions, so that the flow variables are written as

$$
\left(\begin{array}{c}
u(x, y, z, t) \\
v(x, y, z, t) \\
w(x, y, z, t) \\
p(x, y, z, t) \\
c(x, y, z, t)
\end{array}\right)=\left(\begin{array}{c}
0 \\
0 \\
0 \\
p^{(0)}(z) \\
c^{(0)}(z)
\end{array}\right)+\left(\begin{array}{c}
\hat{u}(z, t) \\
\hat{v}(z, t), \\
\hat{w}(z, t) \\
\hat{p}(z, t) \\
\hat{c}(z, t)
\end{array}\right) \exp \left(i a_{x} x+i a_{y} y\right)
$$

where $a_{x}$ and $a_{y}$ are the wavenumbers in the lateral directions. The base state components are denoted by a zero superscript and the quantities $\hat{u}, \hat{v}$, etc are the perturbation amplitudes. Governing equations for the perturbation quantities are obtained by substituting the above quantities into the set of equations (1) and linearizing in the perturbation quantities. The set of linearized equations for the perturbation quantities contain time-dependent coefficients resulting from the assumed form of the gravitational acceleration.

For the linear stability analysis, the problem can be reduced to solving a single fourthorder equation for the the perturbed vertical velocity $\hat{w}$, using the standard manipulation for 
hydrodynamic stability analyses to remove the pressure [15], and the second-order equation for the perturbed solute concentration $\hat{c}$. The variables are made dimensionless by introducing the length scale $D / V$ and the time scale $D / V^{2}$, and by measuring the flow velocity in units of the solidification velocity $V$ and the solute field in units of the far field concentration $c_{\infty}$. The dimensionless linearized equations for the stability problem are then

$$
\begin{gathered}
\frac{1}{S c}\left(L \hat{w}_{t}-L \hat{w}_{z}\right)=L^{2} \hat{w}-a^{2}\left[R s^{(0)}+R s^{(1)} \cos (\Omega t)\right] \hat{c}, \\
\hat{c}_{t}-\hat{c}_{z}+\hat{w} c_{z}^{(0)}=L \hat{c}
\end{gathered}
$$

Here, subscripts indicate partial derivatives, and $c_{z}^{(0)}=-[(1-k) / k] e^{-z}$ is the gradient of the unperturbed solute field. We have defined the operator $L=\left(\partial^{2} / \partial z^{2}-a^{2}\right)$, where $a$ is the spatial wavenumber in the horizontal directions $\left(a=\sqrt{a_{x}^{2}+a_{y}^{2}}\right) . \quad S c=\nu / D$ is the Schmidt number, $R s^{(0)}=g_{0} \beta c_{\infty}(D / V)^{3} /(\nu D)$ is the Rayleigh number based on the mean or d-c gravitational field, $R_{s}{ }^{(1)}=g_{1} \beta c_{\infty}(D / V)^{3} /(\nu D)$ is the Rayleigh number based on the a-c part of the gravitational field, and $\Omega=\bar{\Omega} D / V^{2}$ is the dimensionless oscillation frequency. Table 1 summarizes the dimensional and dimensionless parameters.

The dimensionless boundary conditions at the interface, $z=0$, are

$$
\hat{w}=\hat{w}_{z}=0
$$

and

$$
\hat{c}_{z}+(1-k) \hat{c}=0 .
$$

The perturbations are required to decay as $z \rightarrow \infty$. For numerical purposes, we assume that the spatial domain extends from the interface $z=0$ to a truncated value of the semi-infinite domain denoted by $h_{l}$. The perturbation quantities are set equal to zero at the far-field boundary.

Solutions to the above set of equations which have time-periodic coefficients can be obtained using the framework of Floquet theory [16], in which the solutions are represented as the product of a temporally periodic function and an exponential function of time. Two different numerical implementations of Floquet theory were employed to solve the stability problem formulated above and are discussed briefly below. 
The first approach consists of representing the periodic component of the solutions by a truncated complex Fourier series in time. The solutions for the perturbation quantities $\hat{w}$ and $\hat{c}$ are represented by the product of a periodic Fourier series and an exponential term with complex growth rate $\sigma$,

$$
\begin{aligned}
& \hat{w}(z, t)=e^{\sigma t} \sum_{|m| \leq M} w_{m}(z) e^{i m \Omega t}, \\
& \hat{c}(z, t)=e^{\sigma t} \sum_{|m| \leq M} c_{m}(z) e^{i m \Omega t} .
\end{aligned}
$$

Substitution of the series expansion in to the equations and boundary conditions above yields a set of $12 M+6$ coupled two-point boundary value problems in the spatial variable $z$ for the complex Fourier coefficients $\left(w_{m}, c_{m}\right)$ of the periodic solution components. The resulting set of coupled ordinary differential equations subject to the homogeneous boundary conditions yields an eigenvalue problem that is solved in a manner similar to the one described in [5]. The homogeneous eigenvalue problem is converted into an inhomogeneous. nonsingular problem using the approach suggested by Keller [17]. The coupled set of linear two-point boundary value problems is solved using the computer code SUPORT [18], which uses superposition of numerically integrated solutions with an orthonormalization procedure to maintain the linear independence of the solution set. A high-order Adams-type method is used for the numerical integration of the spatial dependence in the SUPORT code. Here, the temporal resolution depends on the number of coupled Fourier modes $(M)$ used to approximate the periodic part of the solution. The value of $M$ depends most strongly on the modulation frequency, with lower frequencies requiring more temporal modes to obtain a given level of accuracy.

The set of equations contains the complex growth rate $\sigma$ as a parameter. The base state subject to periodic forcing is linearly stable for a given set of parameters if, for all disturbances, $\sigma_{r}<0$, where $\sigma_{r}$ is the real part of $\sigma$. In the calculations, setting $\sigma_{r}=0$ allows for the determination of marginal values of the modulation amplitude, $R s^{(1)}$, and the imaginary part of the growth rate, $\sigma_{i}$, for fixed values of the remaining parameters. For the results presented, only neutral disturbances with $\sigma_{i}=0$ or $\Omega / 2$ are obtained. In this solution 
approach, the number of complex valued unknowns is reduced to $6 M+6$, if the marginal values of $R s^{(1)}$ are determined for the specific values, $\sigma_{i}=0$ or $\Omega / 2$.

The second approach employed consists of approximating the spatial behavior of the disturbance quantities via the pseudospectral technique in the physical domain as described in [19]. The approach corresponds to expanding the solutions in terms of truncated series of Chebyshev polynomials $T_{n}(s)$,

$$
\begin{gathered}
\hat{w}(z, t)=\sum_{n=0}^{N} w_{n}(t) T_{n}(s), \\
\hat{c}(z, t)=\sum_{n=0}^{N} c_{n}(t) T_{n}(s),
\end{gathered}
$$

where $s=\left(2 z / h_{l}\right)-1$. The pseudospectral discretization requires that the solution expansions satisfy the governing equations at specific collocation points for the Chebyshev polynomials. When implemented in the physical domain the unknowns are the solution values at the collocation points. The spatial differential operators in the governing partial differential equations are replaced by discrete matrix operators. As a result, the governing set of partial differential equations and boundary conditions becomes a set of coupled ordinary differential-algebraic equations in time for the unknown solution values at the collocation points.

The computer code DASSL [20] is used to solve the differential-algebraic system for one complete period of the driving temporal modulation. The algorithm uses backward differentiation of up to fifth-order to meet specified local error tolerances. This integration procedure is well-suited for stiff behavior, which is often exhibited by differential-algebraic systems [21]. In this second solution approach, Floquet analysis [16] is implemented by constructing a fundamental solution matrix. The columns of this matrix are linearly independent calculated solutions for the unknowns at the end of one forcing period. The eigenvalues of this matrix are the Floquet multipliers from which the complex growth rate $\sigma$ is obtained. The utility of the second approach is that $\sigma$ itself is the eigenvalue, so that its magnitude is obtained for a given $R s^{(1)}$, which simplifies the search for marginal values and gives more information regarding the proximity of other modes. 
In order to check our numerical results, we have used both methods for selected cases and obtained agreement to five significant figures. Accurate results are obtained for the parameter range studied using from $M=4$ to 8 temporal Fourier modes in the first approach. The required number of modes increases as the frequency decreases. A relative local error tolerance of $10^{-10}$ is used for spatial integration. In the second approach, the Chebyshev series is truncated with $N=20$. A convergence study indicated this number of spatial modes is sufficient for the parameter range studied. For the temporal integration, a relative local error tolerance of $10^{-6}$ was chosen. These same two solution methods were used for a related study of modulated Taylor-Couette flow, where additional details concerning accuracy and convergence are given [22].

As an additional check, the full two-dimensional nonlinear equations were solved using the approach described in [23]. Briefly, the flow field is calculated in two dimensions using the vorticity-streamfunction approach, which eliminates the need to calculate the pressure explicitly. Standard second-order finite-difference techniques are used to solve for the finite amplitude behavior of the two-dimensional velocity and solute concentration fields.

\section{Results and Discussion}

Even for the present simplified model of directional solidification, a significant number of parameters appear in the problem. For the initial calculations, conditions relevant to a microgravity processing environment are assumed. With a background gravitational acceleration of $10^{-6} g_{e}$, the steady-state solutal Rayleigh number $R_{s}{ }^{(0)}$ will typically be very small. It is of interest to determine whether sinusoidal acceleration in time can lead to the onset of solutal convection. So initially, we set $R_{s}{ }^{(0)}=0$, and look for values of $R_{s}^{(1)}$ that lead to instability for given values of the acceleration frequency. Calculations are performed for Schmidt numbers 10 (corresponding to semiconductor type alloys), 81 and 144; the latter two values correspond to the lead-tin and tin-bismuth systems, respectively. The value $k=$ 0.3 is used for the lead-tin system and $k=0.28$ is used for tin-bismuth. The extent of the liquid region, $h_{l}$ is fixed at 15 for all the results presented; this value is sufficiently large that 
it approximates a semi-infinite domain.

In Fig. 2, we show the neutral stability boundaries $\left(\sigma_{r}=0\right)$ at a fixed value of the lateral wavenumber, $a=0.5$, for $S c=10, k=0.3, h_{l}=15$, and $R s^{(0)}=0$. The value $a=0.5$ was chosen, since it is close to the critical value for the unmodulated case. The modulation amplitude, $R s^{(1)}$ is plotted versus the inverse of the modulation frequency. Unstable regions are shaded. For the range of frequencies investigated, two unstable regions are obtained corresponding to growing disturbances exhibiting either synchronous or subharmonic temporal response. At higher frequencies, the the theory predicts unstable subharmonic disturbances, where $\sigma_{i}=\Omega / 2$. The subharmonic stability boundary is lobe-shaped, and approaches infinity as the modulation frequency becomes large. The synchronous stability boundary (where $\left.\sigma_{i}=0\right)$ lies to the right of the subharmonic lobe, but there is a small overlap region. The complete structure of the synchronous region was not investigated. The interest here is in the minimum modulation amplitude that yields instability. It is entirely possible that a banded structure of alternating modes exists above the lowest synchronous boundary; this is a known characteristic of some simpler modulated stability problems [11].

It is interesting to compare the magnitude of the modulation amplitude required to cause instability with the critical solutal Rayleigh number for the unmodulated case. From the results of Hurle et al. [6] for Schmidt number 10, the critical solutal Rayleigh number is 4.43. Thus, for a modulation amplitude of approximately fifteen times the steady critical value, instability resulting from sinusoidal modulation occurs for a range of frequencies. Fig. 3 shows the same type of stability diagram for $S c=81$ with all remaining parameters held fixed. The diagram is qualitatively similar, but both the horizontal and vertical scales have changed substantially. The minimum of the subharmonic branch is larger than the Schmidt number 10 value, and its width is smaller. The dimensional modulation period is $2 \pi D /\left(V^{2} \Omega\right)$; for diffusion coefficients on the order of $10^{-5} \mathrm{~cm}^{2} / \mathrm{s}$ and crystal growth velocities on the order on $10^{-4} \mathrm{~cm} / \mathrm{s}$, a value $\Omega=10$ corresponds to a period of 600 seconds. Our primary interest is in shorter time periods, so we have not extended the calculations to smaller values of $\Omega$.

Results representing the tin-bismuth system, for which space experiments are planned 
[24], are shown in Fig. 4. For this alloy, $S c=144$ and $k=0.28$. The qualitative behavior is the same as Figs. 2 and 3. For this higher Schmidt number, the trend observed for Schmidt number 10 and 81 continues, with the minimum of the subharmonic mode increasing and the width of the lobe decreasing. The reduction of $k$ from 0.3 to 0.28 in this set of calculations has some effect on the magnitude of the modulation amplitude, e.g., with $\Omega=35, R_{s}^{(1)}=$ 570.1 and 517.4 for $k=0.3$ and 0.28 , respectively.

All the results presented so far are for fixed lateral wavenumber. To actually determine the stability of the system, one must find the minimum value of $R_{s}{ }^{(1)}$ for all values of $a$. We have not done this in general due to the extensive number of calculations required. However, for selected frequencies, we have found the minimum as a function of $a$, and these results are shown as the solid dots in Fig. 4. There is only a small difference between the $a=0.5^{\circ}$ values and the minimum values, indicating that fixing $a$ at the value 0.5 is a good approximation.

We have investigated in detail the dependence of $R_{s}{ }^{(1)}$ on $a$ for a fixed $\Omega=32.0$. The results are shown in Fig. 5. There is subharmonic branch at low values of $a$ and a synchronous branch at higher values of $a$, with the two branches intersecting in the vicinity of $a=0.45$. For $a=0.5$, as $R_{s}{ }^{(1)}$ increases the system becomes unstable to a subharmonic disturbance, then regains stability and finally becomes unstable to a synchronous disturbance. The behavior is also evident from Fig. 4 at this frequency. However, when one considers stability with respect to all possible values of $a$, the system is unstable for $R_{s}{ }^{(1)}$ greater than the minimum value on the subharmonic branch, which occurs at $a$ about 0.4 .

The previous results were for $R_{s}(0)=0$, so that the system is stable without modulation. We also consider whether sinusoidal modulation can stablize an otherwise unstable system. As mentioned earlier, the critical Rayleigh number for the unmodulated system is about 5, with a weak Schmidt number dependence [6]. We set $R_{s}{ }^{(0)}=20, \Omega=200$, and $k=$ 0.28 , and calculate the stability of the system for Schmidt numbers of 10 and 144 . The modulation amplitudes, $R s^{(1)}$, as a function of wavenumber, $a$, are shown in Figs. 6 and 7 . For small $R_{s}{ }^{(1)}$, the system is unstable to a synchronous mode. However, as the modulation amplitude increases, the system becomes stable for a range of $R s^{(1)}$, losing stability again to 
a subharmonic mode at sufficiently large values of $R s^{(1)}$. The calculations were limited to $a$ greater than 0.25 . Disturbances with $a$ less than 0.25 correspond to wavelengths greater than $25 \mathrm{D} / \mathrm{V}$, which is on the order of a typical container width. In addition, these disturbance wavelengths are larger than the vertical extent of the domain $\left(h_{l}=15\right)$ which makes the calculations sensitive to the value of $h_{l}$. For sufficiently large values of $a$, the system is stable for zero modulation. The marginal curve $\left(R_{s}{ }^{(0)}\right.$ vs $\left.a\right)$ for the unmodulated case is U-shaped; for $R s^{(0)}=20$, there is a range of $a$ for which the system is unstable. For the cases with modulation shown in Figs. 6 and 7 , the region of instability intersects the axis $R s^{(1)}=0$ (no modulation) in precisely this range. Because the calculations were restricted to $a$ greater than 0.25 , only the upper limit of this range is shown.

To help illustrate the temporal character of the disturbances, we have used the nonlinear code described previously to compute finite amplitude solutions for $\Omega=20$ and three values of $R s^{(1)}$, as shown in Fig. 8. The solutions are computed over nine periods of the gravitational acceleration, and the growth or decay of the solution is examined to compare with the results of the linear analysis shown in Fig. 3. We have plotted the value of the streamfunction $\psi$ at a fixed point in the domain as a function of time for the gravitational acceleration $g$ shown in the lower subplot. For $R s^{(1)}=400$, the system is unstable with a period twice that of the modulation (subharmonic response), in agreement with the prediction of linear theory. If the solutal Rayleigh number is increased to $R s^{(1)}=500$, the system is stable, with a slow decay in the maximum values assumed by the streamfunction over two periods of the modulation. The linear analysis for $R s^{(1)}=500$ shows that the system is linearly stable, with a slowest decaying mode that is subharmonic; there is also a synchronous decaying mode with a decay rate that is three times larger than the subharmonic mode. Finally, for $R s^{(1)}=600$ the system is unstable with a synchronous response, which is also in agreement with the prediction of the linear analysis.

In Fig. 9 we plot the linear eigenfunctions, $\hat{w}(z, t)$ and $\hat{c}(z, t)$, at the onset of instability of the subharmonic mode for $\Omega=20$ and the parameters of Fig. 3. The modulation amplitude, $R s^{(1)}$ is 339.6. The spatial coordinate is normalized by $h_{l}$ and time is normalized by the 
modulation period. Since this is a subharmonic mode, its period is twice the modulation period. The decay of the eigenfunctions as $z \rightarrow 1$ shows that the value of $h_{l}$ used approximates the semi-infinite region well. The eigenfunctions for subharmonic response exhibit the symmetry $\hat{f}(z, t+T)=-\hat{f}(z, t)$, where $T$ is the modulation period.

In summary, in the absence of mean gravitational acceleration $\left(R s^{(0)}=0\right)$ and for large frequencies, instability occurs with subharmonic temporal response to the driving modulation. The amplitude of modulation, $R s^{(1)}$, necessary for instability increases with increasing Schmidt number and modulation frequency. At lower frequencies, instability occurs with synchronous response. When the system is unstable in the absence of modulation, there is a range of modulation amplitudes for which the system is stable.

In the absence of modulation the critical Rayleigh number for the onset of convection is only weakly dependent on the Schmidt number for large Schmidt numbers [6]. However, for the case of pure modulation $\left(R s^{(0)}=0\right)$, it is clear from Figs. 2-4 that $R s^{(1)}$ depends strongly on the Schmidt number.

\section{Acknowledgements}

This work was conducted with the support of the Microgravity Science and Applications Division of the National Aeronautics and Space Administration, and the Applied and Computational Mathematics Program of the Defense Advanced Research Projects Agency. One of the authors (BTM) was supported by a National Research Council Postdoctoral Research Fellowship. 


\section{Table I}

Definition of Symbols

\begin{tabular}{lll}
\hline \multicolumn{2}{c}{ Dimensional Parameters } \\
liquid diffusion coefficient & $D$ & $\mathrm{~cm}^{2} / \mathrm{s}$ \\
solidification velocity & $V$ & $\mathrm{~cm} / \mathrm{s}$ \\
bulk liquid concentration & $c_{\infty}$ & $\mathrm{wt} \%$ \\
kinematic viscosity & $\nu$ & $\mathrm{cm}^{2} / \mathrm{s}$ \\
solutal expansion coefficient & $\beta$ & $\mathrm{wt} \%^{-1}$ \\
vertical domain height & $H$ & $\mathrm{~cm}$ \\
gravitational acceleration & $g(t)=g_{0}+g_{1} \cos (\bar{\Omega} t)$ & $\mathrm{cm} / \mathrm{s}^{2}$ \\
\hline
\end{tabular}

\section{Dimensionless Parameters}

distribution coefficient

$k$

Schmidt number

$$
S c=\nu / D
$$

unmodulated solutal Rayleigh number

$R s^{(0)}=g_{0} \beta c_{\infty}(D / V)^{3} /(\nu D)$

modulated solutal Rayleigh number

$R s^{(1)}=g_{1} \beta c_{\infty}(D / V)^{3} /(\nu D)$

oscillation frequency

$\Omega=\bar{\Omega} D / V^{2}$

vertical domain height

$$
h_{l}=H V / D
$$




\section{References}

[1] W. Knabe and D. Eilers, Acta Astronautica, 9 (1982) 187.

[2] J.I.D. Alexander and C.A. Lundquist, AIAA Journal 26 (1988) 34.

[3] W.S. Liu, M.F. Wolf, D. Elwell and R.S. Feigelson, J. Crystal Growth 82 (1987) 589.

[4] Y.-C. Lu, J.-J. Shiau, R.S. Feigelson and R. K. Route, J. Crystal Growth 102 (1990) 807.

[5] S. R. Coriell, M. R. Cordes, W. J. Boettinger, and R. F. Sekerka, J. Crystal Growth 49 (1980) 13.

[6] D. T. J. Hurle, E. Jakeman, and A. A. Wheeler, Phys. Fluids 26 (1983) 624.

[7] M. E. Glicksman, S. R. Coriell, and G. B. McFadden, Ann. Rev. Fluid Mech. 18 (1986) 307.

[8] J.I.D. Alexander, Microgravity Science and Technology, in press.

[9] S. H. Davis, Ann. Rev. Fluid Mech. 8 (1976) 57.

[10] S. Ostrach, Ann. Rev. Fluid Mech. 14 (1982) 313.

[11] G. Z. Gershuni and E. M. Zhukhovitskii, Convective Stability of Incompressible Fluids (Keter., Jerusalem, 1976) Ch. 8.

[12] P. M. Gresho and R. L. Sani, J. Fluid Mech. 40 (1970) 783.

[13] M. Wadih and B. Roux, J. Fluid Mech. 193 (1988) 391.

[14] S. Biringen and L.J. Peltier, Phys. Fluids A 2 (1990) 754.

[15] S. Chandrasekhar, Hydrodynamic and Hydromagnetic Stability (Dover, New York, 1981) p. 20. 
[16] V. A. Yakubovich and V. M. Starzhinskii, Linear Differential Equations with Periodic Coefficients (Wiley, New York, 1975).

[17] H.B. Keller, Numerical Solutions of Two Point Boundary Value Problems, Regional Conference Series in Applied Mathematics (SIAM, Philadelphia, 1976, Vol. 24).

[18] M.R. Scott and H.A. Watts, SIAM J. Numer. Anal. 14 (1977) 40.

[19] D. Gottlieb, M. Y. Hussaini, and S. A. Orszag, in: Spectral Methods for Partial Differential Equations, Eds. R. G. Voigt, D. Gottlieb, and M. Y. Hussaini (Society for Industrial and Applied Mathematics, Philadelphia, 1984) p. 1.

[20] L. R. Petzold, SAND82-8637, Sandia National Laboratories (1982).

[21] L. R. Petzold, SIAM J. Sci. Stat. Comput. 3 (1982) 367.

[22] B.T. Murray, G.B. McFadden and S.R. Coriell, Phys. Fluids A, submitted for publication.

[23] G.B. McFadden, R.G. Rehm, S.R. Coriell, W. Chuck, and K.A. Morrish, Met. Trans A 15 (1984) 2125.

[24] J.-J. Favier and A. Rouzaud, Advan. Space Res. 6 (1986) 111. 


\section{Figure Captions}

Figure 1. Schematic diagram of directional solidification model.

Figure 2. The modulation amplitude at the onset of solutal convection as a function of the inverse of the frequency for sinusoidal gravitational acceleration.

Figure 3. The modulation amplitude at the onset of solutal convection as a function of the inverse of the frequency for sinusoidal gravitational acceleration.

Figure 4. The modulation amplitude at the onset of solutal convection as a function of the inverse of the frequency for sinusoidal gravitational acceleration. Solid points indicate minimum with respect to wavenumber $a$.

Figure 5. The modulation amplitude at the onset of solutal convection as a function of the wavenumber.

Figure 6. The modulation amplitude at the onset of solutal convection as a function of the wavenumber. In the absence of modulation the system is unstable.

Figure 7. The modulation amplitude at the onset of solutal convection as a function of the wavenumber. In the absence of modulation the system is unstable.

Figure 8. Nonlinear calculations showing the value of the streamfunction at a fixed point in the melt as a function of time. The time-periodic gravitational acceleration is shown in the lower figure. The system is unstable for $R s^{(1)}=400$ and 600 , and is stable for $R s^{(1)}=$ 500.

Figure 9. Linear eigenfunctions $\hat{w}(z, t)$ and $\hat{c}(z, t)$ plotted for two modulation periods (subharmonic response) for $\Omega=20, R_{s}{ }^{(1)}=339.5, R_{s}{ }^{(0)}=0, S c=81$, and $a=0.5$. 


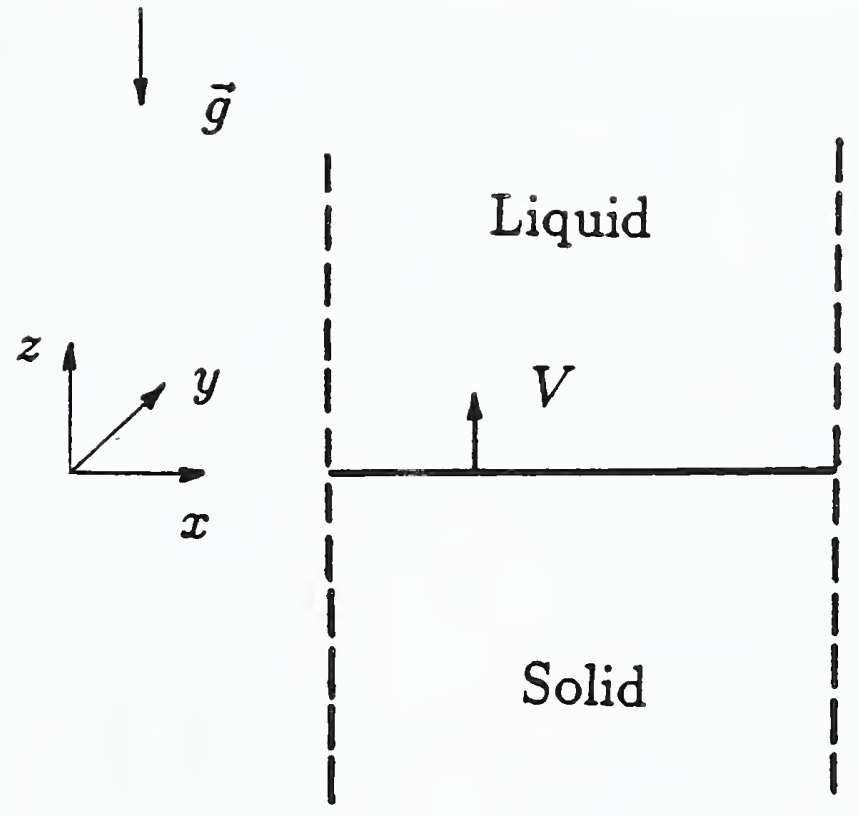

Solute

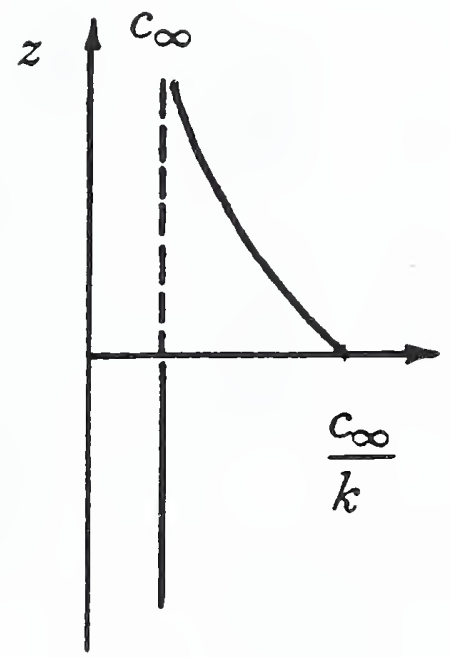


Solutal Convection with Vertical g-Jitter

$$
\begin{gathered}
\mathrm{Sc}=10.0 \quad \mathrm{k}=0.3 \quad \mathrm{~h}_{\iota}=15 \\
\mathrm{RS}^{(0)}=0.0 \quad \mathrm{a}=0.5
\end{gathered}
$$

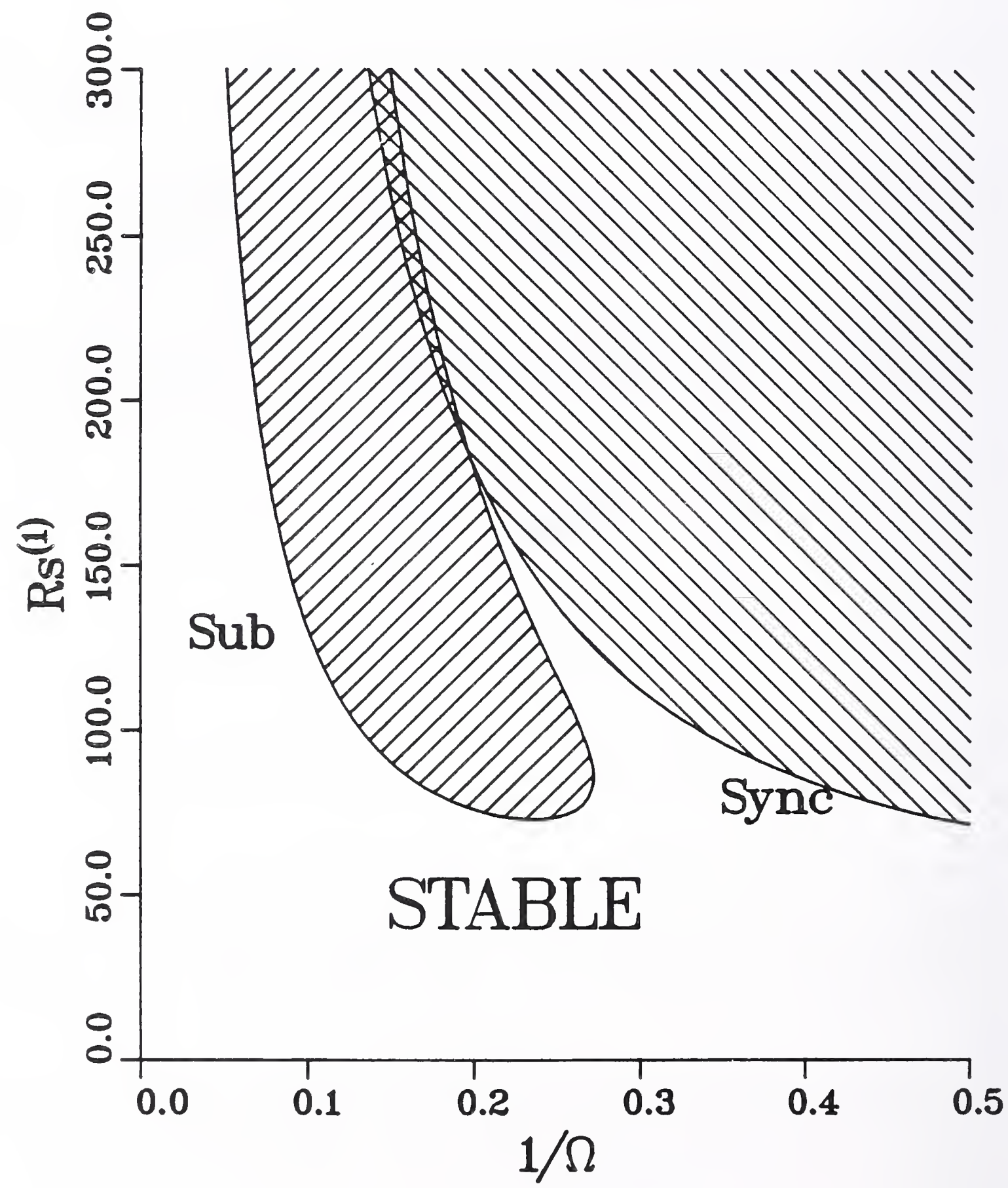


Solutal Convection with Vertical g-Jitter

$$
\begin{gathered}
\mathrm{Sc}=81.0 \quad \mathrm{k}=0.3 \quad \mathrm{~h}_{l}=15 \\
\mathrm{RS}^{(0)}=0.0 \quad \mathrm{a}=0.5
\end{gathered}
$$

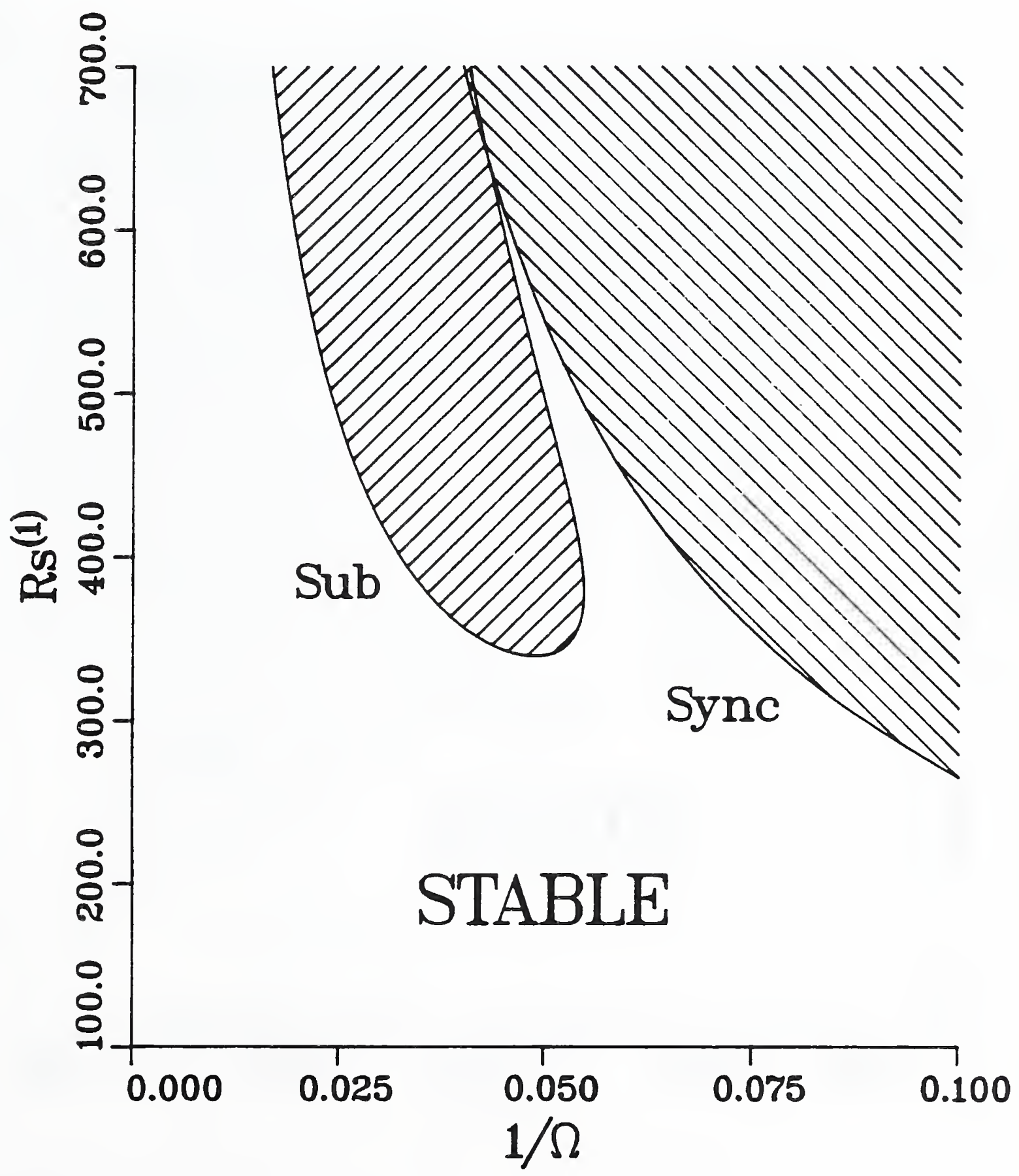


Solutal Convection with Vertical g-Jitter

$$
\begin{gathered}
\mathrm{Sc}=144.0 \quad \mathrm{k}=0.28 \quad \mathrm{~h}_{l}=15 \\
\mathrm{Rs}(0)=0.0 \quad \mathrm{a}=0.5
\end{gathered}
$$

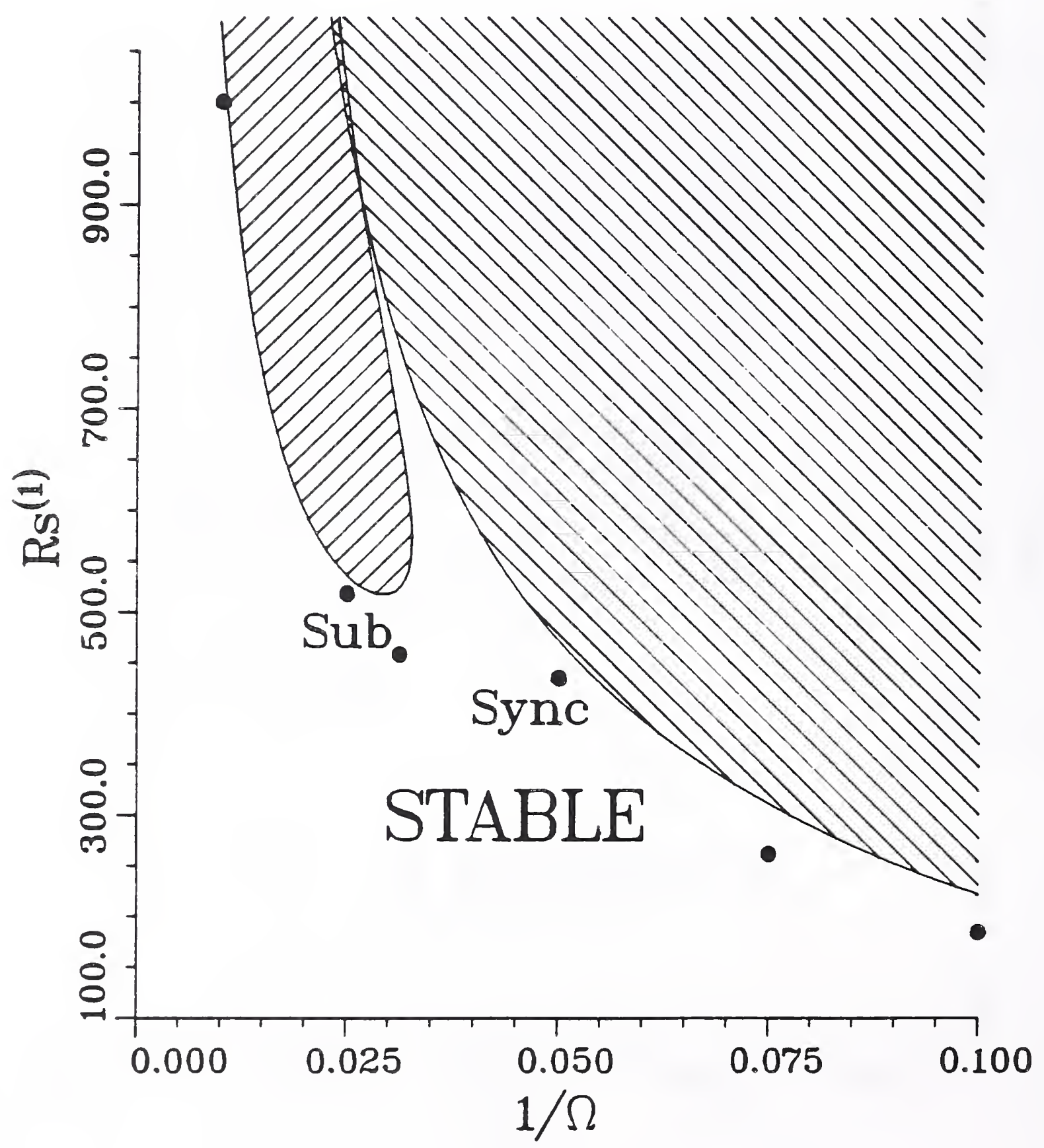

Figure 4 
Solutal Convection with Vertical g-Jitter

$$
\begin{gathered}
\mathrm{Sc}=144.0 \quad \mathrm{k}=0.28 \quad \mathrm{~h}_{l}=15 \\
R \mathrm{~S}^{(0)}=0.0 \quad \Omega=32.0
\end{gathered}
$$

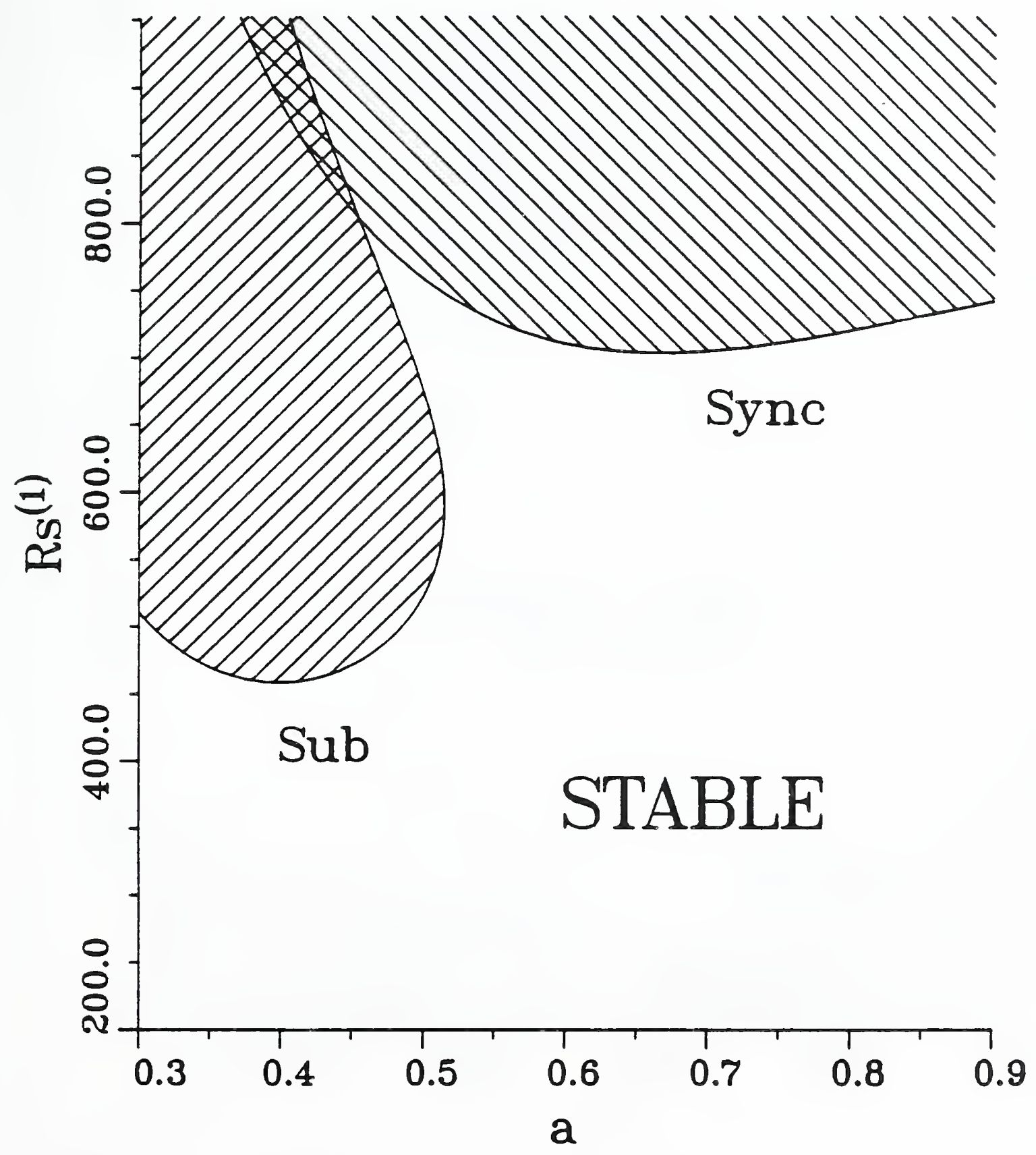


Solutal Convection with Vertical g-Jitter

$$
\begin{gathered}
\mathrm{Sc}=10.0 \quad \mathrm{k}=0.28 \quad \mathrm{~h}_{l}=15 \\
\mathrm{RS}^{(0)}=20.0 \quad \Omega=200.0
\end{gathered}
$$

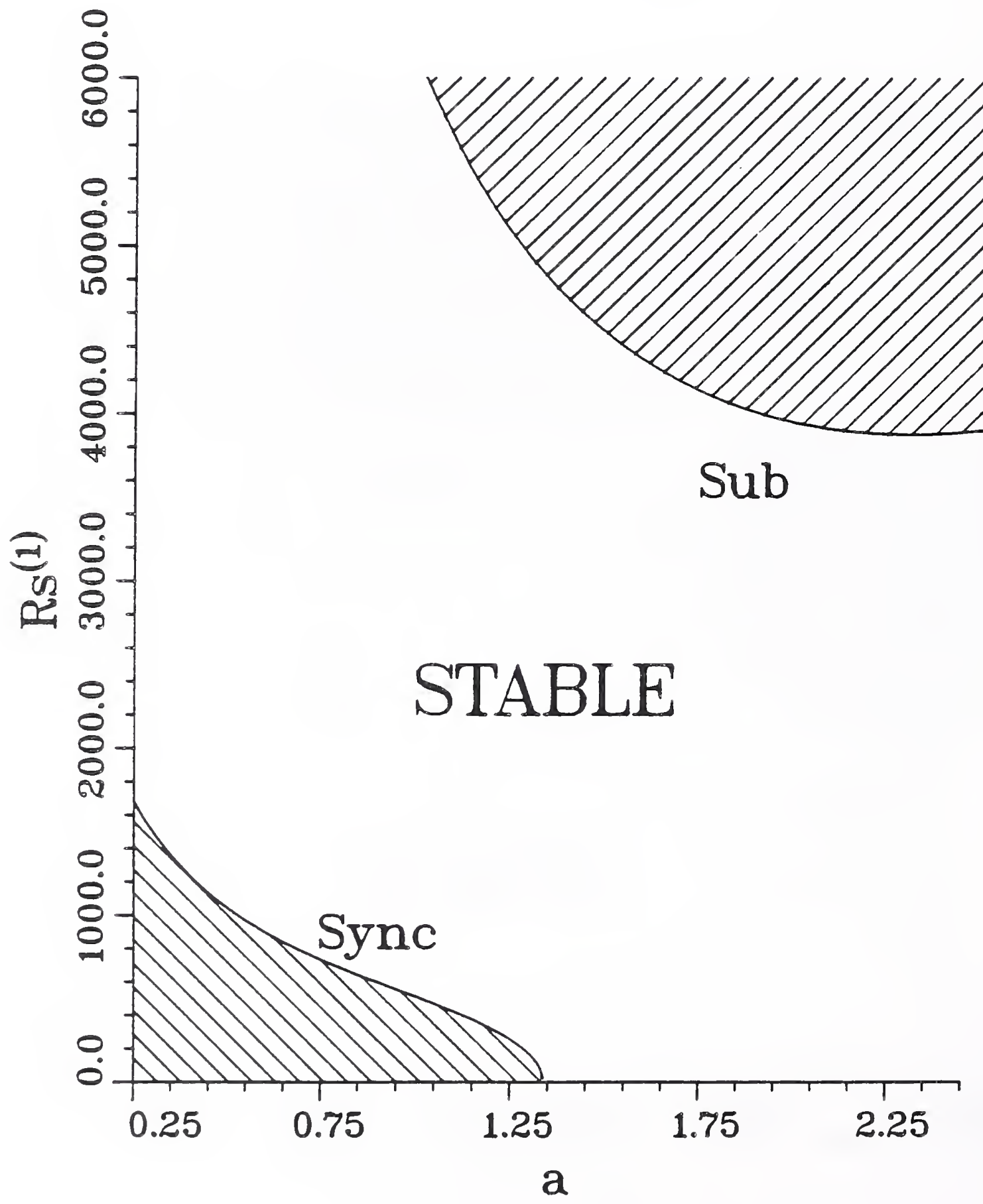

Figure 6 
Solutal Convection with Vertical g-Jitter

$$
\begin{aligned}
& \mathrm{Sc}=144.0 \quad \mathrm{k}=0.28 \quad \mathrm{~h}_{l}=15 \\
& \mathrm{Rs}^{(0)}=20.0 \quad \Omega=200.0
\end{aligned}
$$

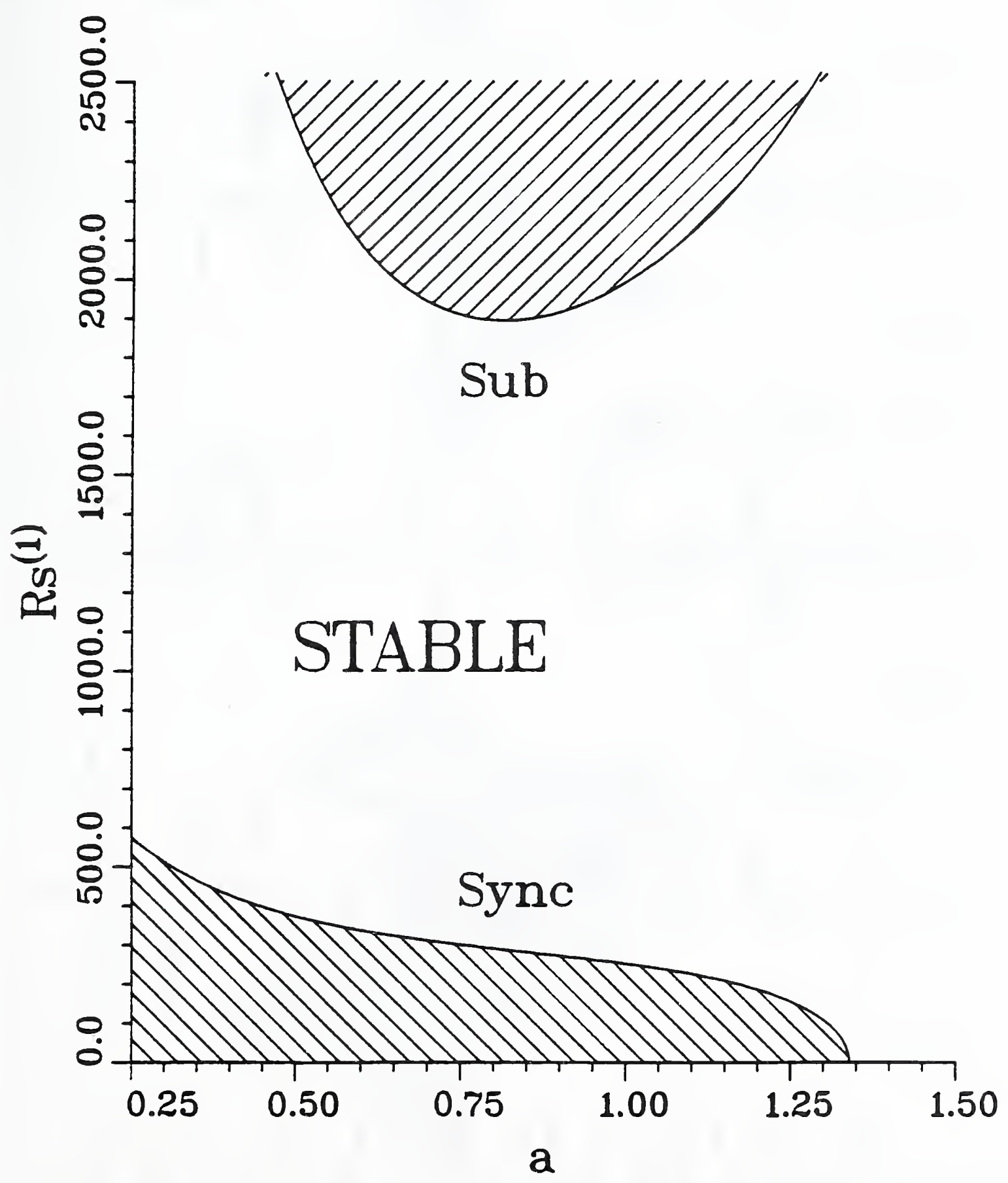

Figure 7 


$$
\begin{array}{ccr}
\mathrm{Rs}^{(0)}=0 & \Omega=20 & \mathrm{~h}_{l}=15 \\
\mathrm{Sc}=81 & \mathrm{k}=0.3 & \mathrm{a}=0.5
\end{array}
$$
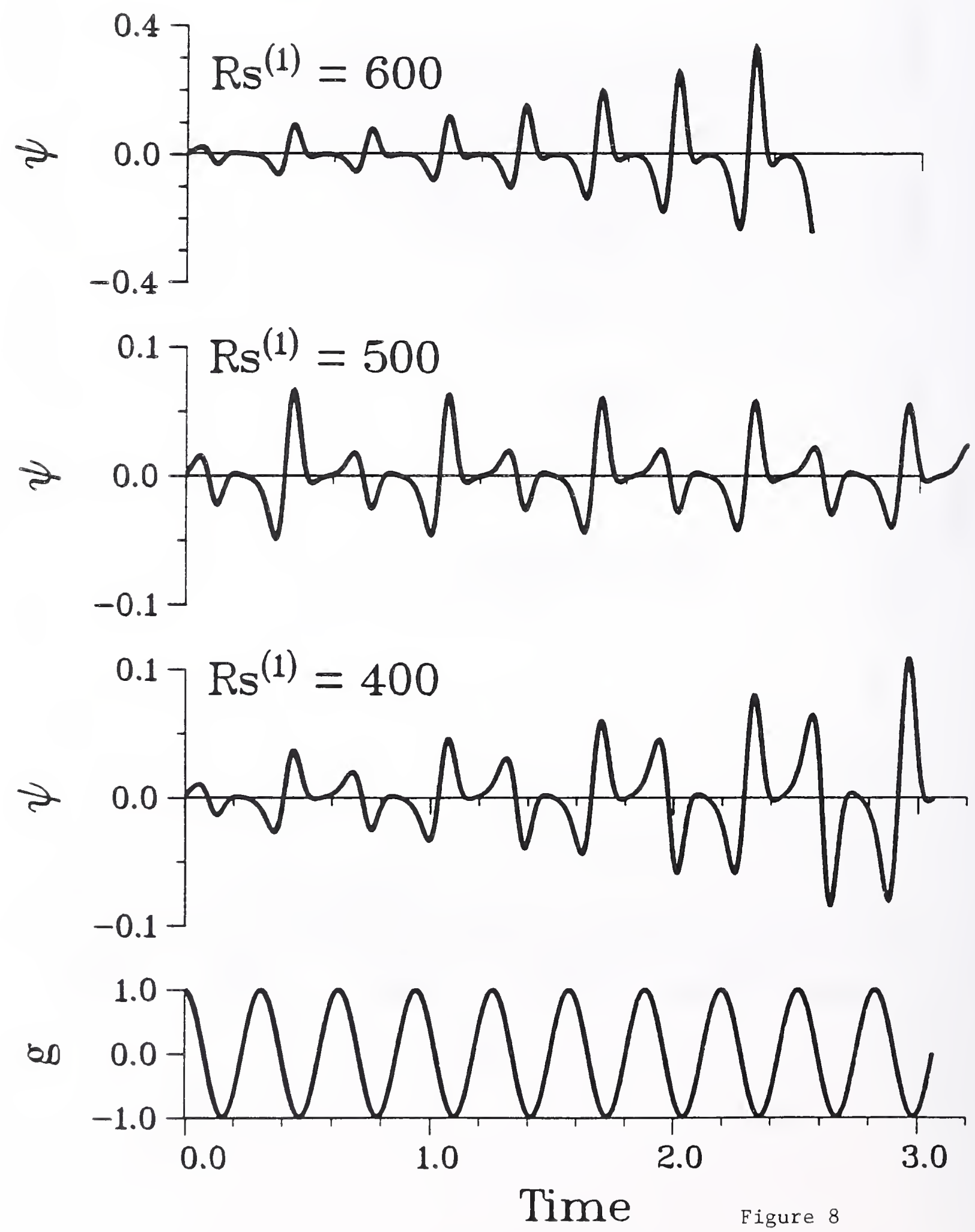

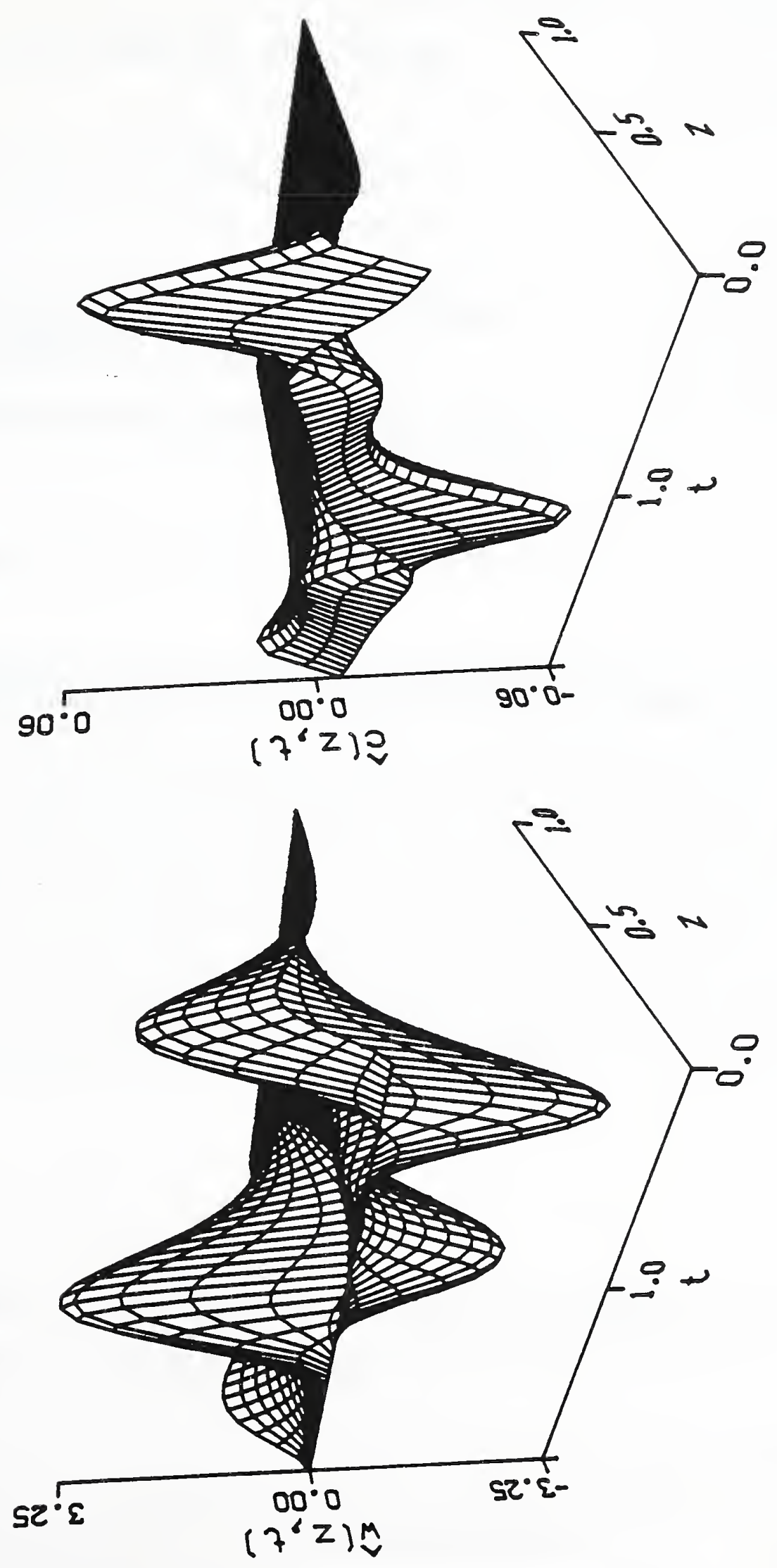

NIST.114A

(REV. 3-89)
U.S. DEPARTMENT OF COMMERCE

NATIONAL INSTITUTE OF STANDARDS AND TECHNOLOGY

BIBLIOGRAPHIC DATA SHEET
1. PULUCATION ON REPOATT NUMEER NISTIR 4389

2 PERFormino OMQANIZATION REPORT NUMBER

3. PUBLCATION DATE

August 1990

4. TITLE AND SUBTITLE

The Effect of Gravity Modulation on Solutal

Convection during Directional Solidification

5. AUTHOR(S)

B. T. Murray, S. R. Coriell, and G. B. McFadden

6. PERFORMING ORGANIZATION (IF JOINT OR OTHER THAN NIST, SEE INSTAUCTIONS)

U.S. DEPARTMENT OF COMMERCE

NATIONAL INSTITUTE OF STANDARDS AND TECHNOLOGY

GATTHERSBURO, MD 20899

7. CONTRACT/OAANT NUMBEA

8. TYPE OF REPORT AND PEAIOD COVERED

9. SPONSORINO ORQANIZATION NAME AND COMPLETE ADDRESS (STAEET, CITY, STATE, ZIP)

10. SUPPLEMENTARY NOTES

DOCUMENT DESCRIBES A COMPUTER PROGRAM; SF-185, FIPS SOFTWARE SUMMARY, IS ATTACHED.

11. ABSTRACT (A 2OO-WORD OA LESS FACTUAL SUMMAAY OF MOST SIGNIFICANT INFOAMATION. IF DOCUMENT INCLUDES A SIONIFICANT BIBUOGRAPHY OR UTERATUAE SURVEY, MENTION IT HERE.)

During directional solidification of a binary alloy at constant velocity, thermosolutal convection may occur due to the temperature and solute gradients associated with the soldification process. For vertical growth in an ideal furnace (lacking horizontal gradients) a quiescent state is possible. For a range of processing conditions, the thermal Rayleigh number is sufficiently small that the stabilizing role of the thermal field during growth vertically upwards may be neglected, and only solutal convection need be considered. The effect of a time-periodic gravitational acceleration (or equivalently vibration) on the onset of solutal convection is calculated based on linear stability using Floquet theory. We find that a stable base state can be destabilized due to modulation, while an unstable state can be stabilized. The flow and solute disturbance fields show both synchronous and subharmonic temporal response to the driving sinusoidal modulation.

12. KEY WOROS (6 TO 12 ENTRIES; ALPHABETICAL ORDER; CAPITAUZE ONLY PROPER MAMES; AND SEPARATE KEY WORDS BY SEMICOLONS)

solutal convection; directional solidification; hydrodynamic stability;

Floquet theory; pseudospectral collocation

13. AVAILABIUTY

X UNUMITED

FOR OFFICIAL DISTRIBUTION. DO NOT RELEASE TO NATIONAL TECHNICAL INFOAMATION SERVICE (NTIS).

OADEA FROM SUPERINTENDENT OF DOCUMENTS, U.S. GOVERNMENT PRINTING OFFICE, WASHINOTON, DC 20402.

OADER FROM NATIONAL TECHNICAL INFORMATION SERVICE (NTIS), SPRINGFIELD, VA 22161.

14. NUMBER OF PRINTED PAGES

30

15. PAICE $\mathrm{AO} 3$ 


